\title{
Safety of low dose methotrexate in elderly patients with rheumatoid arthritis
}

\author{
Boaz Hirshberg, Mordechi Muszkat, Ora Schlesinger, Alan Rubinow
}

\begin{abstract}
Weekly low dose methotrexate is an established treatment for rheumatoid arthritis, but its use in elderly people has not been adequately examined. The aim of this study was to evaluate its safety in elderly patients with rheumatoid arthritis. A retrospective review of the clinical records of rheumatoid arthritis patients over the age of 65 attending a rheumatology unit was conducted. Eligible patients were followed for at least two years and treated with methotrexate in a dose of $7.5 \mathrm{mg} / \mathrm{week}$ while being maintained on concurrent treatment. Thirty three patients were studied. Their mean age was 78.8 years; 32 were female and one was male. Treatment was discontinued in four patients, two because of raised serum liver enzymes and two because of gastrointestinal irritation. No serious adverse events were reported. After two years, haemoglobin levels increased from a mean (SD) of 12.4 (1.3) g/dl to $13.0(1.1) \mathrm{g} / \mathrm{dl}(r=0.226, \mathrm{p}<$ $0.005)$. The white blood count was significantly reduced from $7.9(1.8) \times 10^{9} / 1$ to 6.8 $(1.7) \times 10^{9} / 1(r=0.184, p<0.05)$. No episodes of neutropenia or agranulocytosis were observed. There was a nonsignificant decrease in platelet count. The erythrocyte sedimentation rate decreased from $56.8(30.8)$ to $35.2(24.6) \mathrm{mm} / \mathrm{h}(r=$ $0.246, p<0.01)$. In conclusion, low methotrexate treatment in elderly patients appears to be safe. Routine determination of serum liver enzymes and renal function may reduce individual risk.

(Postgrad Med F 2000;76:787-789)
\end{abstract}

Department of Internal Medicine, Hadassah University Hospital, PO Box 12000 , Jerusalem 91120, Israel

B Hirshberg

Department of Clinical Pharmacology, Hadassah University Hospital

M Muszkat

Rheumatology Unit, Hadassah University Hospital

O Schlesinger

A Rubinow

Correspondence to: Dr Rubinow

rubinov@cc.huji.ac.il

Submitted 20 December 1999

Accepted 9 May 2000
Keywords: methotrexate; elderly; safety

The prevalence of rheumatoid arthritis among people older than 65 years is high, and $30-40 \%$ of patients treated with this disease in rheumatology centres are over 60 years of age. ${ }^{12}$ Methotrexate at a low weekly dose of 7.5 to 20 mg has become an accepted and widely used disease modifying antirheumatic drug for rheumatoid arthritis. ${ }^{3-5}$ While the safety profile of methotrexate under these circumstances is generally good, several studies have shown greater toxicity in elderly patients. ${ }^{6-8}$ It is assumed that reduced renal function may be in part responsible. ${ }^{9}$ Nevertheless, the safety of this treatment in elderly patients has not been established. $^{6}$

Our aim in this study was to evaluate the safety of low dose methotrexate in a cohort of elderly patients with rheumatoid arthritis.
Methods

The clinical medical records of all patients over the age of 65 with rheumatoid arthritis were reviewed retrospectively. The records were identified through a registry of all rheumatoid arthritis patients at the rheumatology unit, Hadassah Medical Centre, Jerusalem. Patients diagnosed with rheumatoid arthritis met the revised criteria of the American Collage of Rheumatology ${ }^{10}$ and were followed for at least two years at three to six month intervals.

The patients were treated with methotrexate at a dose of $7.5 \mathrm{mg} / \mathrm{week}$, given in three doses of $2.5 \mathrm{mg}$ within a 24 hour period. Patients were allowed to continue concurrent drugs such as corticosteroids and non-steroidal antiinflammatory drugs (NSAIDs). During methotrexate treatment, full blood counts-including white blood cell count, haemoglobin, and platelet count-and estimations of liver enzymes (alanine aminotransferase, aspartate aminotransferase, gamma glutamyl transferase, alkaline phosphatase) were performed every month. Measurements of rheumatoid factor and erythrocyte sedimentation rate (ESR) were done every three to six months. A chest $x$ ray was performed every six months.

Medical history and physical examination were verified in each patient. Data were recorded for age, sex, ESR, white blood count, haemoglobin level, platelet count, rheumatoid factor, and liver enzymes.

Changes in laboratory variables obtained during the course of clinical observation were evaluated using of linear regression analysis.

\section{Results}

Thirty three patients were eligible for inclusion in the study. Their mean age was 78.8 years; 32 were female and one was male. Twenty two patients were rheumatoid factor positive and 11 were negative. Two patients discontinued the treatment because of raised liver enzymes. Two further patients decided to stop taking the drug because of gastrointestinal irritation.

Minor adverse events such as oral ulcers were not reported. Serious adverse events such as major infections, hepatic failure, bone marrow depression, inflammatory pneumonitis, or death did not occur.

The hematological variables are summarised in table 1. Haemoglobin levels improved over two years, from a mean (SD) value of 12.4 (1.3) $\mathrm{g} / \mathrm{dl}$ to $13.0(1.1) \mathrm{g} / \mathrm{dl}(r=0.226, \mathrm{p}<$ $0.005)$. The white blood count was significantly reduced from $7.9(1.8) \times 10^{9} / 1$ to 6.8 $(1.7) \times 10^{9} / 1(r=0.184, \mathrm{p}<0.05)$. Neutropenia, agranulocytosis, and thrombocytopenia were not seen. There was a minor decrease in the platelet count, from an average of 324 
Table 1 Haematological follow up over 24 month period

\begin{tabular}{lccrc}
\hline & Baseline & 6 months & 12 months & 24 months \\
\hline Haemoglobin $(\mathrm{g} / \mathrm{dl})$ & $12.4(1.3)$ & $12.7(1.2)$ & $12.9(1.3)$ & $13.0 \ddagger^{\mathrm{a}}(1.1)$ \\
White blood count $\left(\times 10^{9} / \mathrm{l}\right)$ & $7.9(1.8)$ & $7.3(1.7)$ & $7.0(2.0)$ & $6.8^{\star \mathrm{a}}(1.7)$ \\
Platelets $\left(\times 10^{9} / \mathrm{l}\right)$ & $324.4(131.1)$ & $271.8(90.6)$ & $274.0(80.1)$ & $276.3(80.1)$ \\
Erythrocyte sedimentation rate (mm/hour) & $56.8(30.8)$ & $55.2(51.9)$ & $42.0(18.4)$ & $35.2 \dagger^{\mathrm{a}}(24.6)$ \\
\hline
\end{tabular}

Values are mean (SD).

${ }^{\mathrm{a}} \mathrm{p}$ values for $t$ test, significance of differences compared with baseline values.

${ }^{\star} \mathrm{p}<0.05, \dagger \mathrm{p}<0.01, \neq \mathrm{p}<0.005$.

$(131) \times 10^{9} / 1$ to $276(80) \times 10^{9} / 1$, but this was not statistically significant. The ESR decreased over the two years from 56.8 (30.8) to 35.2 (24.6) $\mathrm{mm} / \mathrm{h}(r=0.246, \mathrm{p}<0.01)$.

\section{Discussion}

The prevalence of rheumatoid arthritis among elderly patients is high. ${ }^{12}$ Long term treatment with NSAIDs and corticosteroids carries the risk of osteoporosis, opportunistic infections, and peptic ulcers. Methotrexate is currently considered the standard therapeutic disease modifying antirheumatic drug in patients with rheumatoid arthritis. ${ }^{3-5}$ Large prospective studies have clearly established the efficacy and safety of low dose methotrexate. ${ }^{3}$ However, concern has been raised over the safety of this treatment in elderly patients. ${ }^{6}$ Our present study provides safety data over a two year period in a cohort of 33 elderly patients taking low dose methotrexate. Our results support the view that methotrexate is a safe and useful treatment option in the elderly.

Several large studies have shown that low dose oral pulse methotrexate is an effective and safe treatment for patients with rheumatoid arthritis. Major toxicity is uncommon, while gastrointestinal upset, oral ulceration, transient liver function abnormalities, and neutropeniathough encountered from time to time-are rarely sufficient reasons to discontinue the treatment. ${ }^{7}$ However, most data on the safety profile of methotrexate are limited to younger patients. As only a few elderly patients were included in those studies, data on the safety of methotrexate in the growing number of elderly patients are lacking. ${ }^{6}$ Felson et al, on behalf of the rheumatoid arthritis clinical trial archive group, ${ }^{6}$ attempted to address this issue by pooling data from 11 clinical trials. Of the 496 patients included, 90 were over the age of 65 years. The major determinant of toxicity in their analysis was renal function and not age. A possible limitation of their results is that the patients represented in the trials appeared to be a specially healthy group of elderly people.

Bologna et al assessed the influence of age on the efficacy and toxicity of methotrexate in rheumatoid arthritis. ${ }^{11}$ Four hundred and sixty nine patients were classified according to their age of onset of methotrexate treatment into those who were younger than 65 years $(n=416)$ and older than 65 years $(n=53)$. The frequency and type of side effects were similar. They concluded that age at initiation of methotrexate treatment probably did not influence its efficacy or toxicity in rheumatoid arthritis.

Our cohort represents an unselected group of elderly patients with rheumatoid arthritis treated for two years with low dose methotrexate. An increase in liver enzymes to more than twice the upper limit of normal was found in two patients (six months and one year after the start of treatment). In both of these patients, immediate interruption of treatment caused a return of liver function to normal. In two other patients, the drug was discontinued because of gastrointestinal disturbances. Both these individuals were being treated simultaneously with NSAIDs, which may have caused similar complaints. No neutropenia was reported, and there was no clinical or radiological evidence of inflammatory pneumonitis in any of the patients. Furthermore, the slight decrease in platelet count, the reduction in ESR, and the rise in haemoglobin appear to reflect an improvement in general clinical state.

The methotrexate dose in this study was 7.5 $\mathrm{mg} / \mathrm{week}$, and with this treatment, marked clinical improvement was noted in most of the patients, although the efficacy of treatment was not evaluated directly in this study. As the safety of methotrexate in elderly people has not yet been established, we employed relatively low doses of the drug in our elderly population, although a higher dose is approved for the treatment of rheumatoid arthritis.

The results of our study are limited because of its retrospective nature and the relatively small sample size. We lacked a control group, and more data on clinical efficacy would have been complementary. Unfortunately, prospective data collection was not done. The main goal of this study was to underscore the safety profile of low methotrexate treatment in elderly, as the efficacy of this treatment has already been established. Larger cohorts of elderly patients treated over a long period of time with higher doses of methotrexate are needed to finally establish the safety of this drug.

We conclude that low dose methotrexate treatment in elderly patients appears safe. Simple measures such as follow up of liver and renal function tests and full blood count may eliminate those individuals at risk of unwanted effects.

1 Nesher G, Moore TL. Rheumatoid arthritis in the aged. Incidence and optimal management. Drugs Aging 1993; 3:487-501.

2 Chan KW, Felson DT, Yood RA, et al. Incidence of rheumatoid arthritis in central Massachusetts. Arthritis Rheum 1993;36:1691-6.

3 Weinblatt ME, Maier AL, Fraser PA, et al. Longterm prospective study of methotrexate in rheumatoid arthritis: conclusion after 132 months of therapy. $f$ Rheumatol 1998;25:238-42.

4 Weinblatt ME. Efficacy of methotrexate in rheumatoid arthritis. Br $\mathcal{F}$ Rheumatol 1995;34(suppl):43-8.

5 Buchbinder R, Hall S, Sambrook PN, et al. Methotrexate therapy in rheumatoid arthritis: a life table review of 587 patients treated in community practice. $\mathcal{F}$ Rheumatol patients treated
1993;20:639-44 
6 Rheumatoid Arthritis Clinical Trial Archive Group. The effect of age and renal function on the efficacy and toxicity of methotrexate in rheumatoid arthritis. F Rheumatol
$1995 ; 22: 218-23$.

7 McKendry RJ, Dale P. Adverse effects of low dose methotrexate therapy in rheumatoid arthritis. I Rheumatol 1993;20:1850-6.

8 Tett SE, Triggs EJ. Use of methotrexate in older patients. A risk-benefit assessment. Drugs Aging 1996;9: 458-71.
9 Bressolle F, Bologna C, Kinowski JM, et al. Total and free methotrexate pharmacokinetics in elderly patients with rheumatoid arthritis. A comparison with young patients. $\mathcal{F}$ Rheumatol 1997;24:1903-9.

10 Guidelines for monitoring drug therapy in rheumatoid arthritis. American College of Rheumatology Ad Hoc Committe on Clinical Guidelines. Arthritis Rheum 1996;39:723-31.

11 Bologna C, Viu P, Jorgensen C, et al. Effect of age on the efficacy and tolerance of methotrexate in rheumatoid arthritis. Br f Rheumatol 1996;35:453-7. 